\title{
Radiation-matter transition in Jordan-Brans-Dicke theory
}

\author{
Andrew R. Liddle, Anupam Mazumdar, and John D. Barrow \\ Astronomy Centre, University of Sussex, Falmer, Brighton BN1 9QJ, United Kingdom
}

(Received 12 February 1998; published 25 June 1998)

\begin{abstract}
We study the transition from radiation domination to matter domination in Jordan-Brans-Dicke theory, in particular examining how the Hubble length at equality depends on the coupling parameter $\omega$. We consider the prospects for using high-accuracy microwave anisotropy and large-scale structure data to constrain $\omega$ more strongly than by conventional solar system gravity experiments. [S0556-2821(98)00516-5]
\end{abstract}

PACS number(s): 98.80.Es, 98.65.Dx, 98.80.Cq

\section{INTRODUCTION}

One of the most important epochs in the history of the Universe is the transition from radiation domination to matter domination. This transition alters the growth rate of density perturbations: during the radiation era perturbations well inside the horizon are nearly frozen, but once matter domination commences, perturbations on all length scales are able to grow by gravitational instability. Consequently, the horizon scale at the time of matter-radiation equality is imprinted upon the spectrum of density perturbations; indeed, in a flat cold dark matter cosmology it is the only length scale to appear in the perturbation spectrum. The nature of this transition has been well studied in the standard general relativistic cosmology, and plays a crucial role in calculations of the perturbation spectrum and associated microwave background anisotropies by codes such as CMBFAST [1].

The success of general relativity as a description of our Universe allows us to evaluate the performance of rival theories of gravitation. So far, no weak-field or cosmological observations disagree with the predictions of general relativity. The most important class of deviant theories are scalartensor gravity theories, of which the Jordan-Brans-Dicke (JBD) theory $[2,3]$ is the simplest and best-studied generalization of general relativity. This theory leads to variations in the Newtonian gravitation "constant" $G$, and introduces a new coupling constant $\omega$, with general relativity recovered in the limit $1 / \omega \rightarrow 0$. The most robust constraint on $\omega$, that it must exceed 500, has been derived from timing experiments using the Viking space probe [4], and has stood for nearly 20 years now. Other constraints, such as those from nucleosynthesis [5], are comparable but more model dependent; the most detailed analysis [6] gives only $\omega>50$.

Within the next five to ten years, the advent of new microwave anisotropy satellites, the Microwave Anisotropy Probe (MAP) and Planck, and large galaxy-redshift surveys, 2df and the Sloan Digital Sky Survey (SDSS), promises to revolutionize our understanding of cosmology by permitting the accurate determination of a large number of cosmological parameters [7-9]. So far, estimates of the accuracy of parameter estimation have only been made for cosmological parameters, such as the Hubble constant $H_{0}$ and the matter density $\Omega_{\mathrm{m}}$, and for parameters describing the initial perturbations, such as the spectral index $n$. However, such techniques can also in principle be extended to constrain parameters defining the underlying gravity theory, such as $\omega$, which also influence the gravitational instability process. A full analysis of the viability of obtaining such constraints is an imposing task; all the perturbation formalism employed to compute the present-day matter and radiation power spectra must be generalized to the theory of gravity under consideration, and the results then processed through the Fisher information matrix technology of Refs. [7-9]. In this paper, we assess whether such constraints might be competitive with existing bounds. We do this by studying the properties of the radiation-matter transition in JBD theory. We find that new cosmological data sets may well give limits competitive with those obtained from weak-field solar system tests of general relativity.

\section{EQUATIONS}

The equations for a zero-curvature Friedmann universe are $[2,3,10]$

$$
\begin{gathered}
\left(\frac{\dot{a}}{a}\right)^{2}+\frac{\dot{a}}{a} \frac{\dot{\Phi}}{\Phi}=\frac{\omega}{6}\left(\frac{\dot{\Phi}}{\Phi}\right)^{2}+\frac{8 \pi}{3 \Phi} \rho, \\
\ddot{\Phi}+3 \frac{\dot{a}}{a} \dot{\Phi}=\frac{8 \pi}{2 \omega+3}(\rho-3 p),
\end{gathered}
$$

where the Brans-Dicke coupling, $\omega$, is a constant, $a(t)$ is the cosmological scale factor, and $\Phi(t)$ is the Brans-Dicke field whose present value gives the observed gravitational coupling. Here, $\rho$ and $p$ are the energy density and pressure of the cosmic fluid, which has both matter $\left(p_{\mathrm{m}}=0\right)$ and radiation $\left(p_{\mathrm{r}}=\rho_{\mathrm{r}} / 3\right)$ components; so $\rho=\rho_{\mathrm{m}}+\rho_{\mathrm{r}}$ and $p=p_{\mathrm{r}}$. Assuming negligible energy transfer between the fluids, they still obey the general-relativistic fluid conservation equations with solutions

$$
\rho_{\mathrm{m}}=\rho_{\mathrm{m}, 0}\left(\frac{a_{0}}{a}\right)^{3}, \quad \rho_{\mathrm{r}}=\rho_{\mathrm{r}, 0}\left(\frac{a_{0}}{a}\right)^{4},
$$

where the subscript " 0 " indicates the present value. We set $a_{0}=1$.

We shall assume a spatially flat universe. It is important to realize that since the Brans-Dicke field appears in the Friedmann equation, the corresponding matter density will differ from the general relativity "critical" value, the correction being of order $1 / \omega$, and this must be taken into account in our calculation. In fact, the present density of matter cor- 
responding to a flat universe is higher in JBD theory, as the Brans-Dicke field contributes a negative effective energy density in the Friedmann equation.

The present radiation density has two components. The photon energy density is dominated by the cosmic microwave background, whose measured temperature $T_{\gamma}=2.728$ $\pm 0.004 \mathrm{~K}[11]$ gives

$$
\rho_{\gamma, 0}=4.66 \times 10^{-34} \mathrm{~g} \mathrm{~cm}^{-3} .
$$

However, the standard cosmology also contains relativistic neutrinos. These cannot be directly detected, and so their contribution must be fixed theoretically. Because neutrinos decouple before electron-positron annihilation, they are at a lower temperature than the photons by a factor $\sqrt[3]{4 / 11}$ (see e.g. Ref. [12]), and, assuming three families of massless neutrinos, their total contribution is 0.68 times that of the photons, giving a present total density in relativistic particles of

$$
\rho_{\mathrm{r}, 0}=7.84 \times 10^{-34} \mathrm{~g} \mathrm{~cm}^{-3} .
$$

Accurate modelling of neutrino decoupling [13] may affect this at the percent level, in a calculable way, as might contributions from unknown particles or gravitons decoupling at much higher energies. The redshift of matter-radiation equality is given by

$$
1+z_{\mathrm{eq}}=\frac{\rho_{\mathrm{m}, 0}}{\rho_{\mathrm{r}, 0}} .
$$

Our assumption of spatial flatness fixes the present matter density, where one must be careful to take the contribution of the Brans-Dicke field into account when defining the density parameter, $\Omega$.

\section{A. Analytic approximation}

When one of the fluid components dominates, the attractor solutions are well known [14]. For radiation domination, it is exactly the general relativity solution

$$
a(t) \propto t^{1 / 2}, \quad \Phi=\text { const },
$$

while for matter domination there is a slow variation of the gravitational coupling described by the exact solution ${ }^{1}$

$$
a(t)=\left(\frac{t}{t_{0}}\right)^{(2 \omega+2) /(3 \omega+4)},
$$

\footnotetext{
${ }^{1}$ In general, the JBD cosmologies have exact solutions which show that they are dominated by the $\Phi$ field at early times and by the perfect fluid matter fields at late times. The general solutions approach the exact JBD vacuum solutions as $t \rightarrow 0$ and the particular fluid solutions [given by Eqs. (8)-(12) in the dust case] as $t$ $\rightarrow \infty$ for a flat universe. These attractor solutions also arise as simple exact solutions of Newtonian gravity with $G \propto t^{-n}$ and $a(t) \propto t^{(2-n) / 3}$; see Ref. [15].
}

$$
\begin{gathered}
\Phi=\Phi_{0}\left(\frac{t}{t_{0}}\right)^{2 /(3 \omega+4)}, \\
H_{0}=\left(\frac{2+2 \omega}{4+3 \omega}\right) \frac{1}{t_{0}}, \\
\rho=\frac{\rho_{\mathrm{m}, 0}}{a^{3}}=\left[\frac{3 H_{0}^{2}}{8 \pi G} \frac{(4+3 \omega)(4+2 \omega)}{6(1+\omega)^{2}}\right] \frac{1}{a^{3}}, \\
G=\left(\frac{2 \omega+4}{2 \omega+3}\right) \frac{1}{\Phi_{0}} .
\end{gathered}
$$

Here, Eq. (12) relates the present measured value of the Newton gravitation constant to the $\Phi$ field's value today, the relation being obtained by post-Newtonian expansion [3]. Equation (11) shows how the value of the matter density giving a spatially flat universe is changed from the standard general relativistic expression by a finite $\omega$ value.

We are primarily interested in the Hubble radius at matter-radiation equality, which sets the characteristic scale of the bend in the fluctuation spectrum. It can be estimated analytically by assuming that the matter-dominated solution holds all the way to equality; at equality, both the radiation and matter contribute equally to $\rho$, of course. We solve Eq. (1) with the help of Eqs. (8)-(12) to estimate the Hubble radius. We take only the first-order dependence on $1 / \omega$ in our calculation, which yields

$$
\frac{a_{\mathrm{eq}} H_{\mathrm{eq}}}{a_{0} H_{0}}=\sqrt{2}\left(\sqrt{1+z_{\mathrm{eq}}}\right)(2+\omega) /(1+\omega)\left[1+\frac{0.104}{\omega}\right] .
$$

Using Eqs. (6) and (11), we note that to $O(1 / \omega)$ we have

$$
1+z_{\mathrm{eq}}=24000 h^{2}\left(1+\frac{4}{3 \omega}\right),
$$

where $h$ as usual is the present Hubble constant in units of $100 \mathrm{kms}^{-1} \mathrm{Mpc}^{-1}$, leading to

$$
\frac{a_{\mathrm{eq}} H_{\mathrm{eq}}}{a_{0} H_{0}}=219 h\left[1+\frac{5.81}{\omega}+\frac{\ln h}{\omega}\right] .
$$

The leading term in Eq. (15) is the general-relativistic limit, and is an exact result. Other terms in the equation are the corrections accounting for the variation in $\Phi$ between matter-radiation equality and the present.

\section{B. Numerical approach}

The above result will be accurate at the large $\omega$ values that are of prime interest. However, to obtain the exact behavior, we also tackle the problem numerically.

In addition to the present values of $a, \dot{a}$ and $\Phi$, we also require $\dot{\Phi}$ in order to specify a full solution of the JBD equations. In practice, this additional freedom is eliminated by the attractor behavior during the long radiation-dominated phase. The attractor solution can be picked out in the initial conditions by requiring that $\dot{\Phi} a^{3}$ approach zero for $a$ tending 


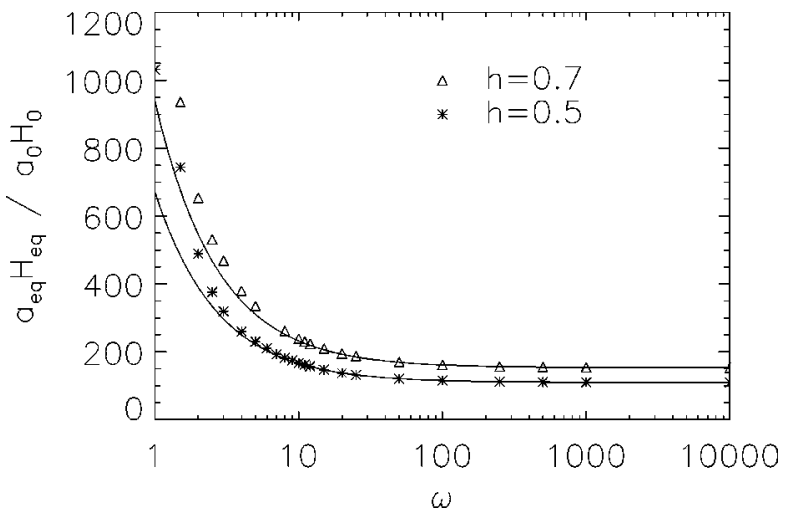

FIG. 1. A comparison of numerical and analytical solutions of the Hubble radius at equality for $h=0.5$ and $h=0.7$. The symbols correspond to the numerical results, and the smooth curves to the analytical estimates of Eq. (15) valid in the large $\omega$ limit.

to zero at the initial singularity [2]. Integrating Eq. (2) once (taking advantage of the radiation contribution canceling out), we obtain

$$
\dot{\Phi} a^{3}=\frac{8 \pi}{2 \omega+3} \rho_{\mathrm{m}, 0} t+C,
$$

and choosing the growing mode amounts to setting the constant $C$ to be zero.

Our numerical code establishes the smooth transition between the radiation- and matter-dominated solutions [Eqs. (7) and (8)]. We study the Hubble length at the transition for all positive values of $\omega$ and plot our numerical output in Fig. 1 , for two different values of $h$. We compare our numerical result with the analytical estimate and confirm our analytical result at the asymptotic limit. At lower values of $\omega$, the analytic approximation becomes inaccurate (due to the neglect of terms of order $\omega^{-2}$ and higher), and underestimates the change in $a_{\mathrm{eq}} H_{\mathrm{eq}}$.

\section{PARAMETER ESTIMATION}

The shift in matter-radiation equality will lead to a shift of the maximum of the power spectrum. In the normal general relativity situation, and assuming all dark matter is cold, this shift is governed by the combination of $\Omega_{\mathrm{m}} h$, where $\Omega_{\mathrm{m}}$ is the matter density parameter, and this is often denoted $\Gamma$. Changing $\Gamma$ gives a horizontal shift to the power spectrum $P(k)$. Other parameters, such as the baryon fraction of the total matter density, also influence the location of the maximum, and sometimes $\Gamma$ is still used as an approximate description of this [16], although future observations are expected to be so good that the baryons have to be included more accurately [17]. Other modifications to the cosmology, such as inclusion of a hot dark matter component, cannot be modelled solely by a shift in the power spectrum.

At the current $95 \%$ lower limit of $\omega=500$, the shift in matter-radiation equality will be at the $1 \%$ level. The parameter $\omega$ can also be probed via its affect on the growth rate of perturbations although, because of biasing, galaxy surveys have problems in constraining the overall normalization.
Note that the lack of a characteristic scale during matter domination means that the altered growth rate will not change the shape of the spectrum; this result holds in JBD theory as well as in general relativity.

Measurement of the shape of the power spectrum from galaxy surveys has been considered in Refs. [8,9]. Tegmark [8] introduces a phenomenological parameter $\eta$ which shifts the power spectrum horizontally, exactly the effect we are interested in, and considers how accurately it can be measured by the SDSS. The accuracy depends on the shortest scales considered; one cannot go too far without worrying about non-linear clustering and biasing effects. If we take Tegmark's estimates evaluated at the scale currently going non-linear $\left(k_{\text {non-lin }} \simeq 0.1 h \mathrm{Mpc}^{-1}\right)$, we can read off the anticipated errors under two assumptions. If all the model parameters are to be determined from the SDSS alone, $\Delta \eta / \eta$ $\simeq 0.1$. If, on the other hand, it is assumed that all parameters are already fixed except $\eta$, then $\Delta \eta / \eta \simeq 0.02$. This is entering at about the required level. At least in principle, data on scales in the non-linear regime can improve this further if their theoretical interpretation is deemed sufficiently unambiguous.

The case where other parameters can be considered as fixed might well apply once one takes microwave background anisotropies into account, and uses them to compute the other cosmological parameters. Indeed, if general relativity is correct, then the Planck satellite alone will already be able to measure $\eta$ at the percent level [8], and then galaxy surveys can be used to improve the estimate further. However, there are many potential degeneracies: the horizon size at equality is changed by altering $\Omega_{\mathrm{m}}$, or $h$, or increasing the number of massless species (perhaps even by including a possible thermal graviton background) as well as by introducing finite $\omega$. On the other hand, the degeneracy may be broken by the different growth rate of perturbations in JBD theory. The evolution of dust density perturbations (where $\delta \equiv \delta \rho / \rho \ll 1$ ) for all wavelengths is determined by the solution of $[18,10]$

$$
\ddot{\delta}+2 \frac{\dot{a}}{a} \dot{\delta}-\frac{8 \pi \rho}{\Phi}\left(\frac{2+\omega}{3+2 \omega}\right) \delta=0 .
$$

Hence, the growing mode solution of Eq. (17) for the background universe of Eqs. (8) and (9) is given by

$$
\delta \propto t^{(4+2 \omega) /(4+3 \omega)} .
$$

This gives a normalization shift at the few percent level for $\omega \sim 500$, most of the effect being after the microwave anisotropies have been generated, since the redshift of decoupling is close to that of equality.

It appears that there is a reasonable prospect that upcoming precision observations can impose a limit on (or make a detection of) finite $\omega$ values at a level competitive with the post-Newtonian bounds, although the use of galaxy surveys for this purpose is subject to a number of caveats given in Ref. [8]. While we have presented the case for arriving at new limits on the constant value of $\omega$ characterizing JBD theories, the basic technique can be extended to constrain the 
value of a non-constant $\omega(\Phi)$ defining cosmological solutions to a more general scalar-tensor gravity theory, as discussed in Ref. [19].

\section{CONCLUSIONS}

We have studied the matter-radiation transition in JBD theory, both numerically and analytically. The shift in the epoch of matter-radiation equality will influence the shape of the density perturbation spectrum, and it appears that precision microwave anisotropy measurements and large galaxyredshift surveys may in the future be able to impose limits on $\omega$ competitive with existing solar system bounds.

However, it seems unlikely that a very substantial improvement will be possible. It may therefore be best to wait to see whether the high quality of data promised is actually delivered before embarking on the substantial undertaking of generalizing general-relativistic results to carry out a proper estimate of the likely observational limits. If all goes well, use of the data to constrain parameters of the gravitational theory will be a worthwhile endeavor and an unexpected bonus from future high-precision observational studies of galaxies and the microwave background.

\section{ACKNOWLEDGMENTS}

A. R. L. is supported by the Royal Society, A. M. by the Inlaks foundation and the ORS, and J. D. B. by PPARC. We thank Ian Grivell, Max Tegmark and Diego Torres for useful discussions, and acknowledge use of the Starlink computer system at the University of Sussex.
[1] U. Seljak and M. Zaldarriaga, Astrophys. J. 469, 1 (1996).

[2] C. Brans and R. H. Dicke, Phys. Rev. 124, 925 (1961); J. D. Barrow, in Current Topics in Astrofundamental Physics, edited by N. Sánchez and A. Zichichi (Kluwer, Dordrecht, 1998), pp. 269-308.

[3] C. M. Will, Theory and Experiment in Gravitational Physics (Cambridge University Press, Cambridge, England, 1993).

[4] R. D. Reasenberg et al., Astrophys. J. 234, L219 (1979).

[5] F. S. Accetta, L. M. Krauss, and P. Romanelli, Phys. Lett. B 248, 146 (1990); J. A. Casas, J. García-Bellido and M. Quirós, Mod. Phys. Lett. A 7, 447 (1992); Phys. Lett. B 278, 94 (1992); D. I. Santiago, D. Kalligas, and R. V. Wagoner, Phys. Rev. D 56, 7627 (1997).

[6] A. Serna, R. Domingues-Tenreiro, and G. Yepes, Astrophys. J. 391, 433 (1992).

[7] G. Jungman, M. Kamionkowski, A. Kosowsky, and D. N. Spergel, Phys. Rev. D 55, 7368 (1996); J. R. Bond, G. Efstathiou, and M. Tegmark, Mon. Not. R. Astron. Soc. 291, L33 (1997); M. Zaldarriaga, D. N. Spergel, and U. Seljak, Astrophys. J. 488, 1 (1997).

[8] M. Tegmark, Phys. Rev. Lett. 79, 3806 (1997).

[9] D. M. Goldberg and M. A. Strauss, Astrophys. J. 495, 29 (1998); M. Tegmark, A. Hamilton, M. Strauss, M. Vogeley, and A. Szalay, ibid., 499, 555 (1998).
[10] S. Weinberg, General Relativity and Cosmology (Wiley, New York, 1972).

[11] D. J. Fixsen, E. S. Cheng, J. M. Gales, J. C. Mather, R. A. Shafer, and E. L. Wright, Astrophys. J. 473, 576 (1996).

[12] E. W. Kolb and M. S. Turner, The Early Universe (AddisonWesley, Redwood City, CA, 1990).

[13] D. Dicus et al., Phys. Rev. D 26, 2694 (1982); N. Y. Gnedin and O. Y. Gnedin, Princeton Report No. astro-ph/9712199; R. E. Lopez, S. Dodelson, A. Heckler, and M. S. Turner, Fermilab Report No. astro-ph/9803095.

[14] H. Nariai, Prog. Theor. Phys. 42, 544 (1969); L. E. Gurevich, A. M. Finkelstein, and V. A. Ruban, Astrophys. Space Sci. 98, 101 (1973).

[15] J. D. Barrow, Mon. Not. R. Astron. Soc. 282, 1397 (1996).

[16] N. Sugiyama, Astrophys. J., Suppl. Ser. 100, 281 (1995).

[17] D. J. Eisenstein and W. Hu, 1997, Princeton Report No. astro-ph/9710252.

[18] H. Nariai, K. Tomita, and S. Kato, Prog. Theor. Phys. 37, 60 (1967).

[19] J. D. Barrow and J. P. Mimoso, Phys. Rev. D 50, 3746 (1994); J. D. Barrow and P. Parsons ibid., 55, 1906 (1997). The general relativistic limit of these scalar-tensor gravity theories requires $\omega(\Phi) \rightarrow \infty$ and $\omega^{\prime}(\Phi) \omega^{-3} \rightarrow 0$. 